\title{
Open-label, add-on trial of cetirizine for neuromyelitis optica
}

Ilana Katz Sand, MD, Michelle T. Fabian, MD, Russell Telford, MAS, Thomas A. Kraus, PhD, Mirna Chehade, MD, MPH, Madhan Masilamani, PhD, Thomas Moran, PhD, Colleen Farrell, MA, CCRC, Shelly Ebel, BSN, Lawrence J. Cook, MStat, PhD, John Rose, MD, and Fred D. Lublin, MD

Neurol Neuroimmunol Neuroinflamm 2018;5:e441. doi:10.1212/NXI.0000000000000441

\section{Abstract}

\section{Objective}

This pilot study preliminarily examined the efficacy and tolerability of cetirizine as an add-on to standard therapy for neuromyelitis optica (NMO).

\section{Methods}

Eligible participants met the Wingerchuk 2006 diagnostic criteria or had a single typical episode along with positive NMO immunoglobulin G. After baseline clinical and laboratory assessments, participants began treatment with cetirizine $10 \mathrm{mg}$ orally daily, in addition to their usual disease-modifying therapy for NMO, and continued for 1 year. The primary end point was the annualized relapse rate (ARR) while on the same disease-modifying therapy before starting cetirizine compared with after taking cetirizine. Additional end points included disability (Expanded Disability Status Scale [EDSS]), relapse severity, tolerability, especially with respect to drowsiness measured by the Epworth Sleepiness Scale (ESS), and laboratory parameters.

\section{Results}

The ARR before cetirizine was $0.4 \pm 0.80$ and after cetirizine was $0.1 \pm 0.24(p=0.047)$. There was no statistically significant difference in the EDSS (mean $3.9 \pm 2.18$ before the start of the study and $3.2 \pm 2.31$ at the conclusion of the study, $p=0.500)$. The ESS remained fairly consistent throughout the study (mean $6.5 \pm 5.33$ at baseline and $6.9 \pm 4.50$ at month $12, p=$ $0.740)$. Laboratory studies were unrevealing.

\section{Conclusions}

In this pilot study, cetirizine was well tolerated, and the prespecified primary efficacy end point was satisfied. However, the open-label design and the small sample size of this pilot study preclude definitive conclusions. Further research is needed.

\section{Classification of evidence}

This study provides Class IV evidence that in patients with NMO, the addition of cetirizine to standard therapy is safe, well tolerated, and reduces relapses.

\author{
Correspondence \\ Dr. Katz Sand \\ ilana.katzsand@mssm.edu
}




\section{Glossary}

AQP4 = aquaporin-4; ARR = annualized relapse rate; EDSS = Expanded Disability Status Scale; ESS = Epworth Sleepiness Scale; ICAM = intercellular adhesion molecule; IgG = immunoglobulin G; IL = interleukin; NMO = neuromyelitis optica; NMOSD = NMO spectrum disorder.

Neuromyelitis optica (NMO) is a rare, severe inflammatory disease that affects the CNS. Neurologic episodes are often severe with poor recovery, resulting in permanent disability. Even with standard treatments, breakthrough disease activity is not uncommon. ${ }^{1}$

Most patients with NMO spectrum disorder (NMOSD) demonstrate autoantibodies to aquaporin-4 (AQP4), a membrane water channel expressed in astrocytes. ${ }^{2}$ Studies suggest that this antibody is pathogenic. Binding of AQP4 immunoglobulin $\mathrm{G}$ (IgG) to AQP4 activates complement-dependent cytotoxicity, which leads to a cascade of inflammatory events including leukocyte infiltration and cytokine release along with breakdown of the blood-brain barrier. Inflammatory damage results in demyelination and axonal/neuronal damage responsible for the observed neurologic deficits. ${ }^{3}$

Research regarding potential therapies initially largely focused on agents that decrease lymphocyte activity. However, animal model work has demonstrated the importance of the local inflammatory events that occur after AQP4-IgG binding. In particular, the importance of eosinophils and their degranulation in the development of NMOSD lesions has been illustrated. Animal model work demonstrated that the development of NMOSD-type lesions could be blocked with the administration of the popular allergy medication, cetirizine, likely related to its properties as an eosinophil stabilizer. ${ }^{4}$

The demonstrated role of eosinophils in the pathogenesis of $\mathrm{NMO}$, effectiveness of cetirizine in an NMO animal model, and favorable safety profile of cetirizine inspired the development of this study, a pilot, open-label, add-on trial of cetirizine for NMO (NCT02865018).

\section{Methods}

\section{Standard protocol approvals, registrations, and patient consents}

The study was approved by the Institutional Review Board of The Icahn School of Medicine at Mount Sinai. Written informed consent was obtained from all participants. The study was registered on clinicaltrials.gov (NCT 02865018).

\section{Clinical protocol initiation}

Eligible participants were 18 years or older and met the Wingerchuk ${ }^{5}$ NMO diagnostic criteria or alternatively had a single characteristic episode along with positive NMO IgG. The disease duration was at least 6 months, and participants were required to be stable on their current disease-modifying therapy for at least 3 months. Those who were already taking a daily antihistamine were excluded, as were those with severe renal or hepatic impairment or known hypersensitivity to cetirizine. Pregnancy and breastfeeding were additional exclusions.

Participants who passed screening completed the baseline clinical assessment. Previous NMOSD history with respect to relapses was captured by a detailed review of medical records. Outside records were obtained when needed. Relapses were defined as "patient-reported symptoms or objectively observed signs typical of an acute inflammatory demyelinating event in the CNS, with duration of at least 24 hours, in the absence of fever or infection," as outlined by the International Panel on the Diagnosis of MS in the 2010 McDonald Criteria ${ }^{6}$ because a rigorous relapse definition for NMOSD was not available in the literature at the time. Each suspected historical relapse identified by the study coordinator was independently reviewed by 2 neurologists (I.K.S. and M.T.F.) with expertise in NMOSD. An additional expert (F.D.L.) was designated to adjudicate cases of disagreement; however, this was not necessary. Participants then underwent standardized neurologic examination (Expanded Disability Status Scale [EDSS] $)^{7}$ and completed the Epworth Sleepiness Scale ${ }^{8}$ (ESS). The ESS asks participants to score on a scale of $0-3$ the likelihood of dozing off/falling asleep during various activities such as watching television and sitting quietly after lunch.

\section{Laboratory assessments}

A blood sample was drawn at baseline. CSF was also sampled in those participants who were amenable. Serum and CSF cytokine analyses included potential biomarkers of NMO, including CXCL $13^{9}$ and IL-21. ${ }^{10}$ Cytokines/chemokines that are associated with eosinophil priming, such as IL-3, IL5, IL-13, granulocyte-macrophage colony-stimulating factor, and $\mathrm{TNF} \alpha,{ }^{11,12}$ or chemotaxis, such as eotaxin, eotaxin-2, eotaxin 3, RANTES, and IL-8, ${ }^{13-16}$ were measured by multiplex ELISA (EMDMillipore, Billerica, MA). In addition, eosinophil-derived neurotoxin was measured by sandwich ELISA as a marker of eosinophil degranulation (MBL laboratories, Nagoya, Japan). Markers of eosinophil activation and survival (CXCR2, CXCR4, CD69, HLA-DR, and intercellular adhesion molecule [ICAM]-1) were analyzed by flow cytometry. Eosinophil activation potential was studied by in vitro stimulation with leukotriene B4 in whole blood culture. ${ }^{17}$

\section{Follow-up assessments}

Participants started cetirizine $10 \mathrm{mg}$ orally daily and were instructed to continue this for 1 year, in addition to their 
established NMOSD disease-modifying therapy. They were monitored for new neurologic episodes and for potential adverse events related to the study drug. Formal relapse assessments as well as the ESS were completed at months 3, 6, 9 , and 12 . The same relapse definition and procedures were applied as with historical relapses, with the additional requirement of in-person evaluation for any suspected relapse. The EDSS was repeated at study conclusion. Blood draw was repeated at month 3 and month 6, and optional CSF was repeated at month 3. Adherence to the study drug was assessed at each visit through discussion with the participant and pill counting for returned bottles.

\section{Statistical analysis}

Participant characteristics were summarized using the median and quartiles or frequencies and percentages. The primary outcome was the difference between the prestudy and on-study annualized relapse rate (ARR). The prestudy ARR was calculated by taking the number of relapses each participant had experienced after the start of their current reported NMO preventative treatment divided by the length of time (in years) they had been receiving this treatment. The on-study ARR was calculated as the number of relapses during the study divided by the length of time in the study. Change in the EDSS was a secondary clinical outcome. Change in the ESS provided a tolerability outcome. Differences in the prestudy vs on-study ARR as well as baseline and month 12 EDSS and ESS were compared using the exact version of the Wilcoxon signed-rank test. Changes in cytokines/chemokines and eosinophil markers were examined graphically, and the differences between visits 1 and 3 were compared using the exact version of the Wilcoxon signed-rank test. All $p$ values for examining changes in cytokines/chemokines were adjusted using the step-down Bonferroni method. Cytokines/chemokines with more than $30 \%$ of the values below detection limits were not examined. These included IL-21, IL-3, IL-5, and eotaxin 3. All analyses were completed using SAS software v9.4 (Cary, NC). The open-label design, small sample size, and lack of control group place all end points under Classification of Evidence Class IV.

\section{Results}

\section{Participant characteristics}

Twenty-four potential participants were referred by treating physicians at the Corinne Goldsmith Dickinson Center for Multiple Sclerosis at Mount Sinai between April 2014 and February 2015. Five participants declined to participate. Three were not eligible because of recent relapse (1), possible plan for pregnancy (1), and multiple medical comorbidities (1). Therefore, sixteen participants were ultimately enrolled. The last participant completed the study in February 2016. Four participants enrolled in the CSF substudy. One participant withdrew before beginning the study medication because her family did not want her to participate.
Baseline demographics and disease characteristics are shown in table. The median age was 36.5 years. Fifteen participants were women. Nine were white and 7 were black. Three selfidentified as Hispanic/Latino. Thirteen historically had at least 1 positive serum NMO IgG. Eight were being treated with rituximab, 7 with mycophenolate, and 1 with azathioprine.

\section{Clinical outcome measures}

Before the study, the patient cohort had experienced a total of 67 relapses. This included 9 events in 7 patients while on the same disease-modifying therapy as at the start of the study. Four patients had 5 relapses in the year before enrollment. Three of these patients (accounting for 3 relapses) remained on the same disease-modifying therapy on entrance to the study. None of the participants changed NMO diseasemodifying therapy during the study period.

During the year-long study period, 1 rituximab-treated participant reported symptoms concerning for a relapse. She developed unilateral pain with eye movements and blurry vision with difficulty distinguishing colors. Examination was notable for a small central scotoma and partial upper field defect as well as color desaturation in the affected eye, although her visual acuity remained normal. Of note, she reported poor adherence to cetirizine in the days leading up to this relapse (3-4 days missed in the week before symptom onset). Two additional participants reported symptoms that led to evaluation for potential relapse. However, in both cases, the treating physician quickly determined that these were related to reemergence of old symptoms based on the history and examination and confirmed with repeat imaging. These events were also reviewed by the appropriate study investigators (I.K.S. and M.T.F.). Figure 1 illustrates these results.

The ARR before cetirizine while participants were treated with the same disease-modifying therapy as at the start of the study was $0.4 \pm 0.80$. With cetirizine as an add-on during the 1-year follow-up period, the ARR was $0.1 \pm 0.24$. This difference reached statistical significance, with a $p$ value of 0.047 . Of note, during data analysis, it was noted that of the 9 events contributing to the prestudy ARR, 3 of them occurred before the 6month mark on the current disease-modifying therapy, the time at which oral therapies are generally recognized to reach full efficacy. If these 3 episodes are removed entirely, there are only 6 events contributing to the prestudy ARR in the year before the start of the study rather than 9 resulting in the loss of statistical significance of the precetirizine vs postcetirizine ARR. The mean EDSS was $3.9 \pm 2.18$ before the start of the study and $3.2 \pm 2.31$ at the conclusion of the study $(p=0.500)$.

\section{Adverse effects and adherence}

Although cetirizine is a second-generation antihistamine, participants were monitored for drowsiness using the ESS as described. The mean ESS was $6.5 \pm 5.33$ at baseline and $6.9 \pm$ 4.50 at month $12(p=0.740)$. Figure 2 shows the ESS over time. The mean ESS at each time point remained consistent 
Table Baseline participant characteristics

\begin{tabular}{|c|c|}
\hline & $\begin{array}{l}\text { Overall } \\
(\mathrm{N}=16)\end{array}$ \\
\hline Age at study enrollment (y): median [Q1, Q3] & $\begin{array}{l}36.5[27.1 \\
51.0]\end{array}$ \\
\hline Age at symptom onset (y): median [Q1, Q3] & $\begin{array}{l}31.0[22.5 \\
41.7]\end{array}$ \\
\hline Female & $15(93.8 \%)$ \\
\hline \multicolumn{2}{|l|}{ Race } \\
\hline Black or African American & $7(43.8 \%)$ \\
\hline White & $9(56.3 \%)$ \\
\hline Hispanic or Latino & $3(18.8 \%)$ \\
\hline Positive NMO antibody in the blood (serum) & $13(81.3 \%)$ \\
\hline \multicolumn{2}{|l|}{ Type of NMO preventative treatment at enrollment } \\
\hline Rituximab & $8(50.0 \%)$ \\
\hline Azathioprine & $1(6.3 \%)$ \\
\hline Mycophenolate & $7(43.8 \%)$ \\
\hline Duration of current treatment (mo): median [Q1, Q3] & $\begin{array}{l}18.3[7.8 \\
40.1]\end{array}$ \\
\hline \multicolumn{2}{|l|}{ No. of previous preventative NMO treatment types } \\
\hline 0 & $10(62.5 \%)$ \\
\hline 1 & $5(31.3 \%)$ \\
\hline 2 & $1(6.3 \%)$ \\
\hline Oral prednisone use at enrollment & $1(6.3 \%)$ \\
\hline Total prestudy relapses: median [Q1, Q3] & $3.0[1.5,5.0]$ \\
\hline $\begin{array}{l}\text { Total prestudy relapses while on current preventative } \\
\text { treatment: median [Q1, Q3] }\end{array}$ & $0.0[0.0,1.0]$ \\
\hline Annualized relapse rate: median [Q1, Q3] & $0.0[0.0,0.7]$ \\
\hline Abbreviation: $\mathrm{NMO}=$ neuromyelitis optica. & \\
\hline
\end{tabular}

throughout the study. There were no other adverse effects reported that were determined to be related to the study drug. Several patients reported improvement in seasonal/ environmental allergies.

The average compliance rate had a median of $92.5 \%$ (interquartile range: $82.7,100 \%$ ). All but 2 participants had an average compliance rate greater than $75 \%$. One of these 2 participants reported that the compliance rate varied from $22 \%$ to $54 \%$ over the course of the study, and the other withdrew from the study before starting the study medication.

\section{Laboratory results}

Laboratory parameters were highly variable without the establishment of clear patterns. There were no clearly measurable significant changes in the parameters tested. These are demonstrated in figure e-1 (http://links.lww.com/ NXI/A23).

\section{Discussion}

Although the treatment of NMOSD with accepted standard disease-modifying therapies such as mycophenolate and rituximab certainly improves patient outcomes, breakthrough disease activity does occur, necessitating continued research regarding additional potential therapies. Current standard treatments have focused on lymphocytes; however, granulocyte infiltration has been shown to play an important role in NMO-related inflammatory destruction. In addition to its antihistaminic functions, cetirizine is known to have eosinophil-stabilizing properties. Cetirizine decreases eotaxinmediated endothelial transmigration ${ }^{18}$ and chemotaxis mediated by platelet-activating factor. ${ }^{19}$ It has been shown to downregulate IL-8 from an epithelial cell line in vitro ${ }^{20}$ and had significant effects on granulocyte infiltration in skin chamber studies after oral administration in allergic patients. ${ }^{21}$ It inhibits IL-5-promoted eosinophil survival ${ }^{22}$ and the adhesion of eosinophils to endothelial cells promoted by IL- $1 .^{23}$ Cetirizine also has a myriad of effects on other immune system components, including inhibition of lymphocyte and monocyte chemotaxis ${ }^{24}$ and various effects on neutrophils, macrophages, and mast cells. ${ }^{25}$ For example, it has been noted to downregulate the expression of ICAM-1, ICAM-3, and lymphocyte function-associated antigen 1 on inflammatory cells in psoriatic skin lesions. ${ }^{26}$ It has also been shown to reduce the number of tryptase-positive mast cells in psoriatic patients. ${ }^{27}$ Tryptase has many effects including inducing the proliferation of type $1 \mathrm{~T}$ helper lymphokines such as IL-1b, IL-6, and TNFa.

Cetirizine's safety has been demonstrated through numerous studies and resulted in over-the-counter status in 2007. The main concern regarding adverse effects in early studies was that it might be sedating. However, previous studies did not find increased drowsiness in atopic individuals or healthy volunteers compared with placebo by either subjective or objective measures. ${ }^{28}$ The previously described effects of cetirizine in allergic diseases combined with the demonstrated role of eosinophils in NMO pathology and potential benefit of cetirizine suggested by NMO animal model work as well as favorable safety profile provided the rationale for this pilot, open-label, add-on trial of cetirizine for NMO.

The prespecified primary end point of reduction in the ARR during the cetirizine study period was satisfied. Even when the 3 episodes that occurred close to the initiation of diseasemodifying therapy are removed and statistical significance is lost, it remains interesting that over the course of 1 year of follow-up of a cohort that included patients with recent breakthrough disease, there was only a single relapse that was quite mild in severity, in a participant who had been poorly adherent to cetirizine.

Caution should be exercised in the interpretation of these results. While a biological effect is plausible, study characteristics such as open-label design and small sample size prohibit 


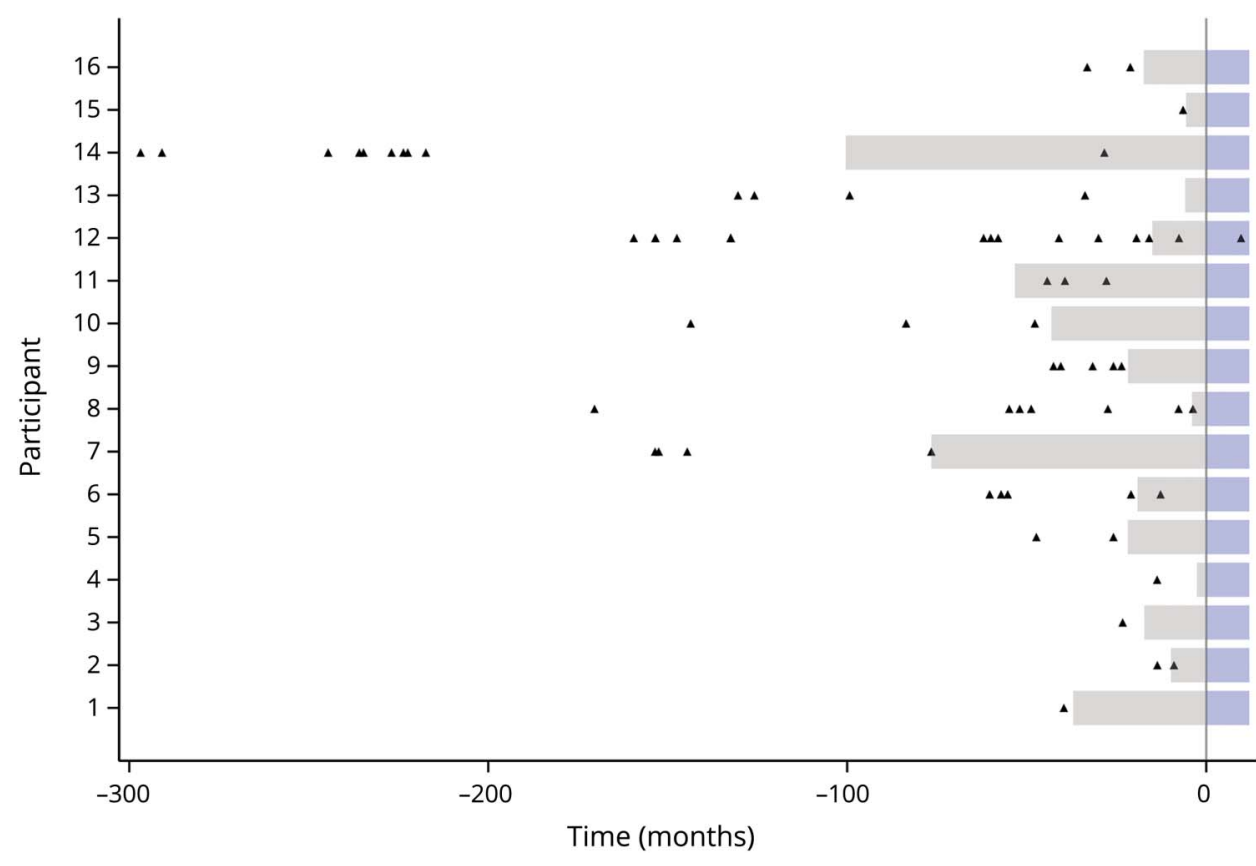

Cetirizine was initiated at time zero. Triangles denote each relapse event. Gray-shaded region = current NMO preventative treatment; blue-shaded region $=$ study period. $\mathrm{NMO}=$ neuromyelitis optica. definitive conclusions. NMO relapses tend to cluster; the requirement for stability for at least 3 months before study entry may have biased the group away from disease activity. As with other prior pilot studies in NMOSD, simple regression to the mean is another possible explanation.

As described, we hypothesized that cetirizine's effects might be mediated through measurable effects on cytokines related to eosinophil activation and migration, given the importance of eosinophils in NMO pathology and cetirizine's demonstrated effects on these pathways in atopic individuals and those with psoriasis. We, therefore, measured serum cytokines and cell adhesion expression markers before and after treatment; however, no statistical differences were observed. There are several potential explanations for this observation. First, approximately half of our participants were also being treated with rituximab, which was given periodically along with high-dose steroids. The timing of blood draws with respect to administration of these medications was not prioritized because of scheduling limitations and likely had an

Figure 2 Epworth Sleepiness Scale recorded throughout the study

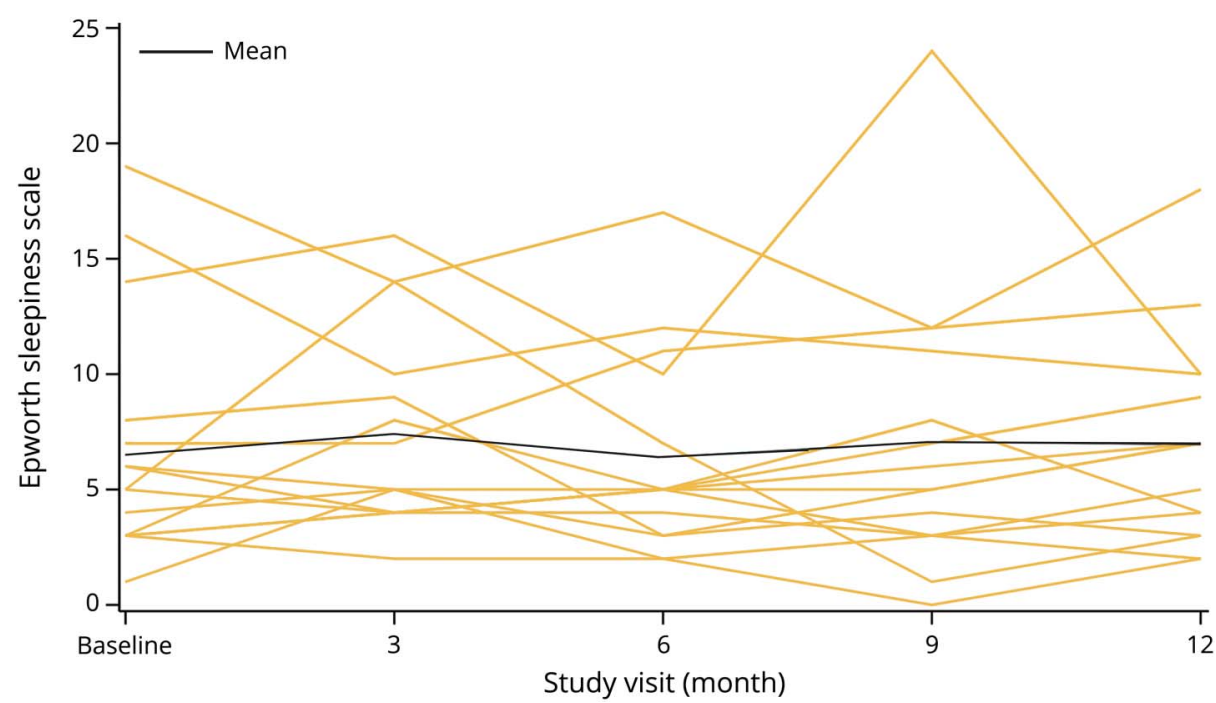

$p=0.740$ from a Wilcoxon signed-rank test of the difference between baseline and month 12 . 
impact on the results. Next, these pathways have not been previously investigated in patients with NMOSD. It is possible that cetirizine's laboratory effects as described in the allergy literature apply only to individuals with active allergic diseases, or that in an NMOSD patient, we would only be able to detect a measurable laboratory effect in a peri-relapse period when eosinophils are in the process of being activated. If this is indeed the case, it is not surprising that we were unable to detect differences in a small number of participants sampled at very few time points, especially since the study required the absence of relapse within 3 months of study entry. Cetirizine given chronically may be able to prevent the activation and chemotaxis of eosinophils and other inflammatory cells, should the beginning of a relapse cascade occur; however, a larger sample size with more frequent specimen collection would be required to determine this definitively.

In our patient population, cetirizine was well tolerated. In particular, no increase in drowsiness was seen. Several participants were pleased with improvement in seasonal allergies.

As noted, by its nature, this pilot study is limited by its small sample size and lack of a control group. Future research directions could include a larger, randomized controlled trial of cetirizine vs placebo as an add-on to standard therapy given on a maintenance basis as in this protocol and/or the incorporation of a larger dose of cetirizine into a regimen to be used at the time of relapse in addition to steroids or plasma exchange.

\section{Author contributions}

The study was conceived by I.K.S. Various aspects of study design were contributed by I.K.S., M.F., F.L., T.A.K., M.M., and M.C. All authors had a role in data collection, analysis, and/or interpretation. Statistical analyses were completed by R.T. and L.C. The manuscript was written by I.K.S. with input from R.T. regarding statistical analyses and T.A.K. regarding laboratory analyses. All authors critically edited the manuscript and approved the final form before submission for publication.

\section{Acknowledgment}

The authors would like to thank the clinicians at the CGD MS Center (Aaron Miller, Stephen Krieger, Sylvia Klineova, Aliza Ben-Zacharia, Gretchen Mathewson) for referring participants and assisting with completion of the trial. They also thank the Guthy Jackson Charitable Foundation for their support as well as the Muzio family for their generous gift that allowed completion of all aspects of the study. Most importantly, they thank their NMO patients for their participation.

\section{Study funding}

The study was funded by a research grant from the Guthy Jackson Charitable Foundation to I.K.S. Supplemental funds were provided through a philanthropic grant by the Muzio family to Mount Sinai.

\section{Disclosure}

I. Katz Sand received research support from the US Department of Defense, the National Multiple Sclerosis Society, and the Guthy Jackson Charitable Foundation. M.T. Fabian served on the speaker's bureau of Biogen. R. Telford reports no disclosures. T.A. Kraus received research support from the $\mathrm{NIH}$ and receives royalty payments from Mount Sinai. M. Chehade consulted for Shire and Actelion and received research support from Shire, Regeneron, Allakos, and Nutricia. M. Masilamani has been employed by Boehringer Ingelheim Pharmaceuticals Incorporation. T. Moran served on the editorial board of the Journal of Virology. J. Rose received research support from Biogen, Teva Neuroscience, VA Merit Review Grant, the National Multiple Sclerosis Society, the Guthy Jackson Charitable Foundation, and Friends of MS. C. Farrell, S. Ebel, and L.J. Cook report no disclosures. F.D. Lublin served on the scientific advisory board of, consulted for, and received travel funding from Bayer Healthcare Pharmaceuticals, Biogen, EMD Serono, Novartis, Teva Neuroscience, Actelion, Sanofi-Aventis, Acorda, Roche/Genentech, Celgene, Genzyme, MedImmune, Receptos, Forward Pharma, Akros, TG Therapeutics, AbbVie, Toyama, MedDay, Atara Biotherapeutics, Mapi, Innate, Polpharma, and Apitope. He is a co-chief editor of Multiple Sclerosis and Related Disorders; served on the speaker's bureau of Genzyme and Roche/ Genentech; received research support from Biogen Idec, Teva, the NIH, Acorda, Biogen Idec, Novartis, Sanofi Genzyme, Celgene, and NMSS; and has provided expert testimony. Full disclosure form information provided by the authors is available with the full text of this article at Neurology.org/NN.

Received November 6, 2017. Accepted in final form December 12, 2017.

\section{References}

1. Mealy MA, Wingerchuk DM, Palace J, Greenberg BM, Levy M. Comparison of relapse and treatment failure rates among patients with neuromyelitis optica: multicenter study of treatment efficacy. JAMA Neurol 2014;71:324-330.

2. Lennon VA, Kryzer TJ, Pittock SJ, Verkman AS, Hinson SR. IgG marker of opticspinal multiple sclerosis binds to the aquaporin-4 water channel. J Exp Med 2005;202: 473-477.

3. Ratelade J, Verkman AS. Neuromyelitis optica: aquaporin-4 based pathogenesis mechanisms and new therapies. Int J Biochem Cel Biol 2012;44:1519-1530.

4. Zhang H, Verkman AS. Eosinophil pathogenicity mechanisms and therapeutics in neuromyelitis optica. J Clin Invest 2013;123:2306-2316.

5. Wingerchuk DM, Lennon VA, Pittock SJ, Lucchinetti CF, Weinshenker BG. Revised diagnostic criteria for neuromyelitis optica. Neurology 2006;66:1485-1489.

6. Polman CH, Reingold SC, Banwell B, et al. Diagnostic criteria for multiple sclerosis: 2010 revisions to the McDonald criteria. Ann Neurol 2011;69:292-302.

7. Kurtzke JF. Rating neurologic impairment in multiple sclerosis: an expanded disability status scale (EDSS). Neurology 1983;33:1444-1452.

8. Johns MW. A new method for measuring daytime sleepiness: the Epworth Sleepiness Scale. Sleep 1991;14:540-545

9. Alvarez E, Piccio L, Mikesell RJ, et al. CXCL13 is a biomarker of inflammation in multiple sclerosis, neuromyelitis optica, and other neurological conditions. Mult Scler 2013;19:1204-1208.

10. Wu A. Cerebrospinal fluid IL-21 levels in neuromyelitis optica and multiple sclerosis. Can J Neurol Sci 2012;39:813-820.

11. Lampinen M, Carlson M, Hakansson LD, Venge P. Cytokine-regulated accumulation of eosinophils in inflammatory disease. Allergy 2004;59:793-805.

12. Carlson M, Peterson C, Venge P. The influence of IL-3, IL-5, and GM-CSF on normal human eosinophil and neutrophil C3b-induced degranulation. Allergy 1993;48: 437-442.

13. Ponath PD, Qin S, Ringler DJ, et al. Cloning of the human eosinophil chemoattractant, eotaxin. Expression, receptor binding, and functional properties suggest a mechanism for the selective recruitment of eosinophils. J Clin Invest 1996;97: 604-612. 
14. Sehmi R, Cromwell O, Wardlaw AJ, Moqbel R, Kay AB. Interleukin-8 is a chemoattractant for eosinophils purified from subjects with a blood eosinophilia but not from normal healthy subjects. Clin Exp Allergy 1993;23:1027-1036.

15. Banwell ME, Tolley NS, Williams TJ, Mitchell TJ. Regulation of human eotaxin-3/ CCL26 expression: modulation by cytokines and glucocorticoids. Cytokine 2002;17: 317-323.

16. Kameyoshi Y, Dorschner A, Mallet AI, Christophers E, Schroder JM. Cytokine RANTES released by thrombin-stimulated platelets is a potent attractant for human eosinophils. J Exp Med 1992;176:587-592.

17. Takafuji S, Tadokoro K, Ito K, Nakagawa T. Release of granule proteins from human eosinophils stimulated with mast-cell mediators. Allergy 1998;53:951-956.

18. Thomson L, Blaylock MG, Sexton DW, Campbell A, Walsh GM. Cetirizine and levocetirizine inhibit eotaxin-induced eosinophil transendothelial migration through human dermal or lung microvascular endothelial cells. Clin Exp Allergy 2002;32: 1187-1192.

19. Fadel R, David B, Rassemont R, Herpin-Richard N, Borgnon A, Rihoux JP. Eosinophil infiltration: effects of $\mathrm{Hl}$ antihistamines. J Am Acad Dermatol 1991:24:1094-1096.

20. Arnold RR. Cetirizine counter-regulates interleukin-8 release from human epithelial cells (A549). Clin Exp Allergy 1999;29:1681-1691.
21. Charlesworth ENE. Effect of cetirizine on mast cell-mediator release and cellular traffic during the cutaneous late-phase reaction. J Allergy Clin Immunol 1989;83:905-912.

22. Sedgwick JB, Busse WW. Inhibitory effect of cetirizine on cytokine-enhanced in vitro eosinophil survival. Ann Allergy Asthma Immunol 1997;78:581-585.

23. Kyan-Aung U, Hallsworth M, Haskard D, De Vos C, Lee TH. The effects of cetirizine on the adhesion of human eosinophils and neutrophils to cultured human umbilical vein endothelial cells. J Allergy Clin Immunol 1992;90:270-272.

24. Jinquan T, Reimert CM, Deleuran B, Zachariae C, Simonsen C, Thestrup-Pedersen K. Cetirizine inhibits the in vitro and ex vivo chemotactic response of $\mathrm{T}$ lymphocytes and monocytes. J Allergy Clin Immunol 1995;95:979-986.

25. Namazi MR. Cetirizine and allopurinol as novel weapons against cellular autoimmune disorders. Int Immunopharmacol 2004;4:349-353.

26. Pestelli EE. Cetirizine modulates adhesion molecule expression in a double-blind controlled study conducted in psoriatic patients. Int J Tissue React 2003;25:1-8.

27. Pestelli EE. Cetirizine reduces the number of tryptase-positive mast cells in psoriatic patients: a double-blind controlled study. Int J Tissue React 2001;23:97-103.

28. Campoli-Richards DM, Buckley MM, Fitton A. Cetirizine. A review of its pharmacological properties and clinical potential in allergic rhinitis, pollen-induced asthma, and chronic urticaria. Drugs 1990;40:762-781. 


\section{Neurology \\ Neuroimmunology \& Neuroinflammation}

Open-label, add-on trial of cetirizine for neuromyelitis optica

Ilana Katz Sand, Michelle T. Fabian, Russell Telford, et al.

Neurol Neuroimmunol Neuroinflamm 2018;5;

DOI 10.1212/NXI.0000000000000441

This information is current as of February 2, 2018

\section{Updated Information \& Services}

References

Citations

Subspecialty Collections

Permissions \& Licensing

Reprints including high resolution figures, can be found at: http://nn.neurology.org/content/5/2/e441.full.html

This article cites 28 articles, 2 of which you can access for free at: http://nn.neurology.org/content/5/2/e441.full.html\#\#ref-list-1

This article has been cited by 1 HighWire-hosted articles: http://nn.neurology.org/content/5/2/e441.full.html\#\#otherarticles

This article, along with others on similar topics, appears in the following collection(s):

All Clinical trials

http://nn.neurology.org//cgi/collection/all_clinical_trials All Demyelinating disease (CNS)

http://nn.neurology.org//cgi/collection/all_demyelinating_disease_cns Devic's syndrome

http://nn.neurology.org//cgi/collection/devics_syndrome

Information about reproducing this article in parts (figures,tables) or in its entirety can be found online at:

http://nn.neurology.org/misc/about.xhtml\#permissions

Information about ordering reprints can be found online: http://nn.neurology.org/misc/addir.xhtml\#reprintsus

Neurol Neuroimmunol Neuroinflamm is an official journal of the American Academy of Neurology.

Published since April 2014, it is an open-access, online-only, continuous publication journal. Copyright Copyright $\odot 2018$ The Author(s). Published by Wolters Kluwer Health, Inc. on behalf of the American Academy of Neurology.. All rights reserved. Online ISSN: 2332-7812.

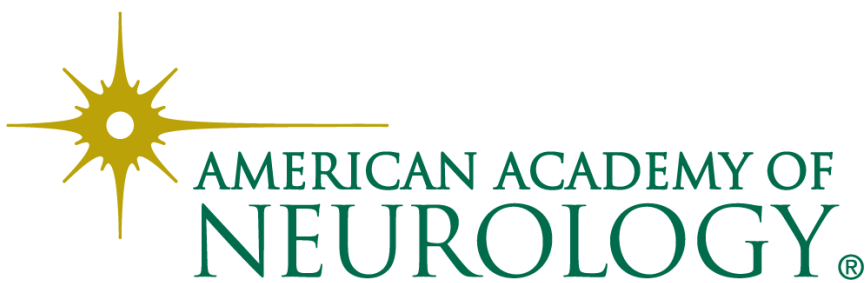

